\title{
Baicalin reduces the severity of experimental autoimmune encephalomyelitis
}

Y. Zeng ${ }^{1,3}$, C. Song ${ }^{1}$, X. Ding ${ }^{1}, \mathrm{X} . \mathrm{ji}^{2}$, L. $\mathrm{Yi}^{4}$ and $\mathrm{K}$. Zhu ${ }^{4}$

\author{
${ }^{1}$ Department of Neurology, The First Affiliated Hospital, \\ ${ }^{2}$ Department of Immunology, ${ }^{3}$ Department of Neurology, \\ The Second Affiliated Hospital, Nanjing Medical University, Nanjing, China \\ ${ }^{4}$ Department of Neurology, General Hospital of PLA, Beijing, China
}

\author{
Correspondence \\ C. Song \\ Department of Neurology \\ 300 Guangzhou Road \\ Nanjing, 210029 \\ China \\ Fax: +86-25-8367-4680 \\ E-mail: ayingzeng@hotmail.com \\ $\ldots \ldots \ldots \ldots \ldots \ldots \ldots$
}

Received October 17, 2006 Accepted March 26, 2007

\begin{abstract}
Scutellaria baicalensis Georgi is one of the important medicinal herbs widely used for the treatment of various inflammatory diseases in Asia. Baicalin (BA) is a bioactive anti-inflammatory flavone found abundantly in Scutellaria baicalensis Georgi. To explore the therapeutic potential of BA, we examined the effects of systemic administration of the flavone (5 and $10 \mathrm{mg} / \mathrm{kg}$, ip) on relapsing/remitting experimental autoimmune encephalomyelitis (EAE) induced by proteolipid protein 139-151 in SJL/J mice, an experimental model of multiple sclerosis. The mice treated with PBS or BA at day -1 and for 3 consecutive days were observed daily for clinical signs of disease up to 60 days after immunization. In the PBS-EAE group, neurological scores were: incidence (100\%), mean day of onset $(8.0 \pm 0.73)$, peak clinical score (3.0 \pm 0.4$)$, and cumulative disease index (141.8 \pm 19.4 ). In the BA-EAE group (5 or $10 \mathrm{mg} \mathrm{kg}^{-1} \mathrm{day}^{-1}$, respectively), incidence ( 95 or $90 \%$ ), mean day of onset $(9.0 \pm 0.80$ or $9.2 \pm 0.75$; $\mathrm{P}=0.000$ ), peak clinical score $(2.2 \pm 0.3$ or $2.0 \pm 0.3 ; \mathrm{P}=0.000)$, and cumulative disease index $(75.9 \pm 10.1$ or $62.9 \pm 8.4 ; \mathrm{P}=0.000)$ decreased, accompanied by the histopathological findings (decrease of dense mononuclear infiltration surrounding vascellum) for the spinal cord. Additionally, the in vitro effects of $\mathrm{BA}(5,10$, and $25 \mu \mathrm{M})$ on mononuclear cells collected from popliteal and inguinal lymph nodes of day-10 EAE mice were evaluated using an MTT reduction assay for cell proliferation, and ELISA to measure IFN- $\gamma$ and IL- 4 cytokines. Compared with the control group, BA caused an increase in IL-4 (EAE-DMSO: $3.56 \pm 0.42 \mathrm{pg} / \mathrm{mL}$ vs EAE-BA $(5,10$, and $25 \mu \mathrm{M}$ ): 6.03 $\pm 1.1,7.83 \pm 0.65,10.54 \pm 1.13 \mathrm{pg} / \mathrm{mL}$, respectively; $\mathrm{P}<0.001$ ); but inhibited IFN- $\gamma$ (EAE-DMSO: $485.76 \pm 25.13 \mathrm{pg} / \mathrm{mL} v s$ EAE-BA (5, 10 , and $25 \mu \mathrm{M}$ ): $87.08 \pm 9.24,36.27 \pm 5.44,19.18 \pm 2.93 \mathrm{pg} / \mathrm{mL}$, respectively; $\mathrm{P}<0.001)$ and the proliferation of mononuclear cells (EAE-DMSO: $0.73 \pm 0.021 v s$ EAE-BA $(5,10$, and $25 \mu \mathrm{M}): 0.41 \pm$ $0.015,0.31 \pm 0.018,0.21 \pm 0.11$, respectively; $\mathrm{P}<0.001)$ in a concentration-dependent manner. The results suggest that BA might be effective in the treatment of multiple sclerosis.
\end{abstract}

\section{Key words}

- Experimental autoimmune encephalomyelitis

- Baicalin

- IFN- $\gamma$

- IL-4

- Neurologic score 


\section{Introduction}

Experimental autoimmune encephalomyelitis (EAE) is a CD4+ $4^{+}$-cell-mediated inflammatory demyelinating disease of the central nervous system (CNS) that is commonly used as a model of multiple sclerosis (MS). The highest incidence of MS onset is between 20 and 40 years. The etiology of MS is unclear, but it is considered to be the result of the perivascular migration of activated T-cells and mononuclear cells into the CNS where they persist and induce an inflammatory cascade that results in injury to previously normal neural tissue (1). Th1 cytokines such as IFN- $\gamma$ and TNF- $\alpha$, and Th2 cytokines such as IL-4 are believed to play a key role in this process. In view of the wide variety of cytokines and chemokines involved in inflammatory and autoimmune diseases, strategies that modulate the activity of multiple cytokines may prove useful.

Scutellaria baicalensis Georgi (Labiatae) is one of the important medicinal herbs for the treatment of cancer, viral diseases and inflammatory diseases in East Asian countries. It has been reported to contain a large number of flavonoids, among them the flavone baicalin (5,6-dihydroxy-4-oxygen-2phenyl-4H-1-benzopyran-7-beta-D-glucopyranose acid, BA). Scutellaria radix is yet another plant rich in BA. BA is known for its anti-inflammatory and smooth muscle relaxant properties and as a constituent of some hepatoprotective herbal mixtures such as Xiaochahu-tang (Sho-saiko-to), popular in Japan and China (2-4). Previous studies have shown that $100 \mu \mathrm{g} / \mathrm{mL} \mathrm{BA}$ inhibits by $98 \%$ in vitro superantigenic staphylococcal exotoxin-stimulated $\mathrm{T}$-cell proliferation and production of IL- $1 \beta$, TNF- $\alpha$, IFN- $\gamma$ by peripheral blood mononuclear cells, a Th1type immune response $(5,6)$.

The present study was designed to examine the efficacy of BA in abrogating EAE induced by proteolipid protein 139-151 $\left(\mathrm{PLP}_{139-151}\right)$ in mice and to elucidate the pos- sible mechanism involved.

\section{Material and Methods}

\section{Animals}

Female SJL/J mice (8-10 weeks of age; Jackson Laboratory, Bar Harbor, ME, USA) were housed in the Laboratory Animal Center of Nanjing Medical University. The research was conducted in accordance with the internationally accepted principles for laboratory animal use and care. The Scientific Committee of the First Affiliated Hospital of Nanjing Medical University approved all experimental protocols that involved the use of animals. All mice were age matched for individual experiments and were housed in groups with a 12-h light/dark cycle. The access to food and water was facilitated for paralyzed mice to prevent dehydration.

\section{Proteolipid protein peptide and baicalin}

Immunodominant mouse PLP $_{139-151}$ peptide (HSLGKWLGHPDKF) was synthesized by Genemed synthesis (South San Francisco, CA, USA), purity was assessed by HPLC $(>97 \%)$. It was initially solubilized in LPSfree phosphate-buffered saline (PBS) to obtain a concentration of $4 \mathrm{mg} / \mathrm{mL}$ and stored at $-80^{\circ} \mathrm{C}$. BA was provided by the National Institute for the Control of Pharmaceutical and Biological Products (Beijing, China), purity was assessed by HPLC (>95\%). Molecular formula: $\mathrm{C}_{21} \mathrm{H}_{18} \mathrm{O}_{11}$, molecular weight: 446.36, and CAS registry number: 21967-41-9. The structure is shown in Figure 1.

\section{Induction of experimental autoimmune encephalomyelitis}

Mice were immunized subcutaneously in the left flank at three sites with a total of $0.15-\mathrm{mL}$ emulsion containing $150 \mathrm{mg}$ $\mathrm{PLP}_{139-151}$ and an equal volume of complete 
Freund's adjuvant (Sigma-Aldrich, St. Louis, MO, USA). Immediately and $48 \mathrm{~h}$ later, 300 ng pertussis toxin (Sigma-Aldrich) was injected subcutaneously. The mice $(\mathrm{N}=20$, per group) were treated with PBS or BA (5 and 10 $\mathrm{mg} \mathrm{kg}^{-1}$ day $^{-1}$, respectively, in PBS, with $\mathrm{pH}$ adjusted to 7.2) beginning on the day -1 of the immunization and for 3 consecutive days. Control mice $(\mathrm{N}=12)$ treated with PBS were injected with complete Freund's adjuvant plus pertussis toxin according to the same schedule (data not shown).

\section{Clinical evaluation}

Mice ( $\mathrm{N}=20)$ were observed daily for clinical signs of disease up to 60 days after immunization. Mice were scored according to their clinical severity as follows: grade 0 , no abnormality; grade 0.5 , partial loss of tail tonicity, assessed by inability to curl the distal end of the tail; grade 1, reduced tail tone of slightly clumsy; grade 2, tail atony, moderately clumsy gait, impaired righting ability, or any combination; grade 3 , hind limb weakness of partial paralysis; grade 4, complete hind limb paralysis or fore limb weakness; grade 5, tetraplegia or moribund state; grade 6 , death (7). The data were plotted as daily mean clinical score for all animals in a particular treatment group. Several parameters of disease were examined to evaluate the severity of EAE and the efficacy of BA therapy that include mean clinical score, incidence, mean day of onset, peak clinical score, and cumulative disease index (8).

\section{Histopathology}

The lumbar region of the spinal cord was dissected from mice $(\mathrm{N}=8$, per group) at the peak of clinical disease between 14-20 days after immunization and fixed in $10 \%$ formalin. The spinal cords were cut into $4-\mu \mathrm{m}$ thick sections after fixation and embedded in paraffin before sectioning. The sections were stained with hematoxylin and eosin (Sigma-Aldrich) for histologic analysis. Histologic scores were graded on each crosssection, according to the following criteria: 0 , no infiltrate; 1 , three or less focal meningeal infiltrates; 2 , more than three focal meningeal infiltrates; 3 , up to five perivascular infiltrate foci in the parenchyma; 4, five to ten perivascular foci in the parenchyma; 5 , more than ten perivascular foci or diffuse infiltration.

\section{Cell proliferation assay and cytokine analysis}

Mice ( $\mathrm{N}=10$, per group) were killed at day 10 after PLP $_{139-151}$ sensitization. The popliteal and inguinal lymph nodes (PILN) were removed, dissected and dispersed through a stainless steel wire mesh as described (9). Cells were then washed three times in complete modified Eagle's medium (Flow, Irvine, UK) supplemented with modified RPMI-1640 medium containing $25 \mathrm{mM}$ HEPES, L-glutamine (Gibco, Paisley, UK), $100 \mathrm{U} / \mathrm{mL}$ penicillin, $100 \mathrm{mg} / \mathrm{mL}$ streptomycin (Gibco), and 10\% (v/v) heat-inactivated FCS (Gibco). PILN mononuclear cell (MNC) was adjusted to an appropriate density for analysis.

BA was dissolved in dimethylsulfoxide (DMSO) as $10 \mathrm{mM}$ stock solutions and diluted to $1 \mathrm{mM}$ with culture medium just before use. Blank cells received an equivalent amount of DMSO. The final concentration of DMSO did not exceed $0.2 \%(\mathrm{v} / \mathrm{v})$.

An MTT (Sigma-Aldrich; $2.5 \mathrm{mg}$ of MTT/

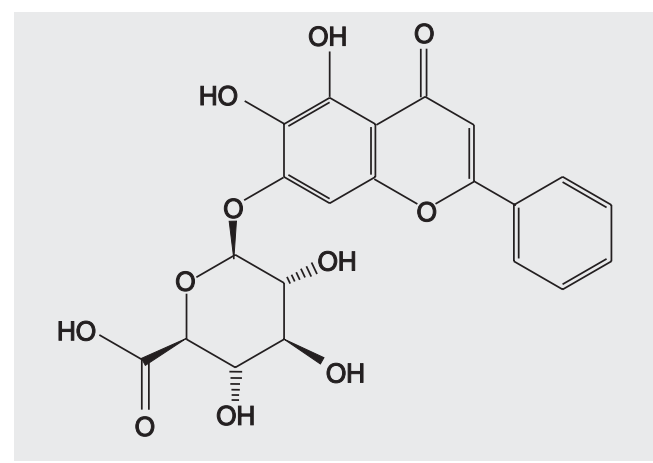

Figure 1. The structure of baicalin. 
$\mathrm{mL}$ in PBS) assay (10) was performed to measure the proliferation of PILN MNC. Aliquots of $100 \mu \mathrm{L}$ PILN MNC suspensions from EAE and control mice were cultured in triplicates in round-bottomed 96-well polystyrene microtiter plates (Nunc, Copenhagen, Denmark) at a cell density of $2 \times 10^{6}$ cells $/ \mathrm{mL}$ in culture medium. PILN MNC were stimulated for $72 \mathrm{~h}$ with $\mathrm{PLP}_{139-151}$ and treated for $72 \mathrm{~h}$ with various concentrations of BA or an equivalent amount of medium containing DMSO at $37^{\circ} \mathrm{C}$ in $95 \%$ humidity and $5 \% \mathrm{CO}_{2}$ (3 wells for each sample). Blank cells from control mice received an equivalent amount of medium containing DMSO. After drug treatments (final concentration: $100 \mu \mathrm{g} / \mathrm{mL} \mathrm{PLP}_{139-151} ; 5,10$, and $25 \mu \mathrm{M} \mathrm{BA}$ ) the supernatant was removed, MTT was dissolved in PBS to a concentration of $5 \mathrm{~g} / \mathrm{L}$, and $20 \mu \mathrm{L}$ was added to each well of a 96well plate. The MTT-containing medium was then removed after 4-h incubation, DMSO was added to dissolve the reduced formazan product. Finally, the plate was read at $550 \mathrm{~nm}$ with a microplate reader (Bio-Rad 2550, Richmond, CA, USA).

One $\mu \mathrm{g} / \mathrm{mL}$ PILN MNC suspensions from EAE and control mice were added to each well of round-bottomed 24-well polystyrene microtiter plates (Nunc) at a cell density of 5 x $10^{6}$ cells $/ \mathrm{mL}$ in culture medium. Each treatment as described above was performed in triplicate. PILN MNC were stimulated with $100 \mu \mathrm{g} / \mathrm{mL}$ PLP $_{139-151}$ for $72 \mathrm{~h}$ and treated with various concentrations of BA or an equivalent amount of DMSO for $72 \mathrm{~h}$. Culture supernatant was collected and stored at $-70^{\circ} \mathrm{C}$ until use. Cytokines (IFN- $\gamma$ and IL4) were detected in culture supernatants on the same day with the same reagent using commercially available Mouse ELISA kits (Diaclone Research, Besancon, France) according to manufacturer instructions. Absorbance was read at $450 \mathrm{~nm}$ using an enzyme-linked immunosorbent microplate reader (Bio-Rad; Model 2550). Standard curves for each assay were generated and the concentration of the cytokines in the cell supernatants was determined by extrapolation from the appropriate standard curve. The lower limits of detection for each assay were $15 \mathrm{pg} / \mathrm{mL}$ for IFN- $\gamma$ and $3.0 \mathrm{pg} / \mathrm{mL}$ for IL-4. The intra-assay coefficient of variation was $<5 \%$ for IFN- $\gamma$ and IL-4.

\section{Statistical analysis}

Analyses were performed using one-way ANOVA and Kruskal-Wallis ANOVA with post hoc analysis using Dunnett's multiple comparison test, or Fisher's exact test, as appropriate. Results are reported as mean \pm $\mathrm{SD}$; P values $<0.05$ were considered to be statistically significant.

\section{Results}

\section{Effects of baicalin on the clinical signs of mice}

We evaluated the ability of BA (5 and 10 $\mathrm{mg} / \mathrm{kg}$, respectively) to affect induction and progression of EAE after immunization with $\mathrm{PLP}_{139-151}$. Treatment with BA (5 or $10 \mathrm{mg}$ $\mathrm{kg}^{-1}$ day $^{-1}$ ) was initiated prior to immunization (from day -1 to day 1 relative to immunization) for 3 consecutive days. BA ( 5 or 10 $\mathrm{mg} \mathrm{kg}{ }^{-1}$ day $^{-1}$ ) led to a statistically significant reduction in EAE clinical score. These effects led to significant clinical improvement and delayed disease progression during the following 60 days of observation, indicating that BA inhibited both development and progression of EAE (Table 1 and Figure 2).

\section{Effect of baicalin on inflammation in the spinal cord}

The neuropathological changes in EAE and MS are associated with the blood-brain barrier breakdown and infiltration by MNCs. In order to identify the mechanism of therapeutic efficacy in EAE, we studied the effect 
of BA on the inflammation in the CNS in EAE model. Spinal cord from BA-treated mouse shows lesser dense mononuclear infiltration surrounding vascellum and edge compared with that from PBS-treated mouse $(\mathrm{P}=0.000)$ (Figure 3).

\section{Effects of baicalin treatment on peripheral T-cell responses}

PILN MNCs from SJL/J mice with EAE were analyzed for proliferation and cytokine responses to the immunizing PLP $_{139-151}$ peptide. Immune cell responses were assessed just after disease onset (day 10). As shown in Figure 4A, BA inhibited proliferation responses to $\mathrm{PLP}_{139-151}$ peptide in a concentra-
Table 1. Effect of treatment with baicalin on neurological impairment during experimental autoimmune encephalomyelitis (EAE) in SJL/J female mice.

\begin{tabular}{lcccc}
\hline Group & $\begin{array}{c}\text { Incidence } \\
\text { (number/total) }\end{array}$ & $\begin{array}{c}\text { Mean day of } \\
\text { onset }\end{array}$ & $\begin{array}{c}\text { Peak clinical } \\
\text { score }\end{array}$ & $\begin{array}{c}\text { Cumulative } \\
\text { disease index }\end{array}$ \\
\hline PBS/EAE & $20 / 20(100 \%)$ & $8.0 \pm 0.73$ & $3.0 \pm 0.4$ & $141.8 \pm 19.4$ \\
BA (5 mg/kg) & $19 / 20(95 \%)$ & $9.0 \pm 0.80^{*}$ & $2.2 \pm 0.3^{*}$ & $75.9 \pm 10.1^{*}$ \\
BA (10 mg/kg) & $18 / 20(90 \%)$ & $9.2 \pm 0.75^{*}$ & $2.0 \pm 0.3^{*}$ & $62.9 \pm 8.4^{*}$
\end{tabular}

Female SJL/J mice were immunized with proteolipid protein 139-151 (day 1) and treated intraperitoneally with baicalin (BA) (5 or $10 \mathrm{mg} / \mathrm{kg}$ ) (day -1 to day 1 ) as described in Material and Methods. Incidence of disease evaluated by Fisher's exact test $\left(\chi^{2}=2.105, P=0.349\right)$. Mean day of onset and clinical score were evaluated by the Kruskal-Wallis test with post hoc analysis using Dunnett's multiple comparison test.

${ }^{*} \mathrm{P}=0.000$ compared to phosphate-buffered saline (PBS). Cumulative disease index was determined as the sum of daily scores for each mouse in the indicated group compared to PBS and the data were analyzed by ANOVA ( ${ }^{*} \mathrm{P}=0.000$ with post hoc analysis using Dunnett's multiple comparison test).

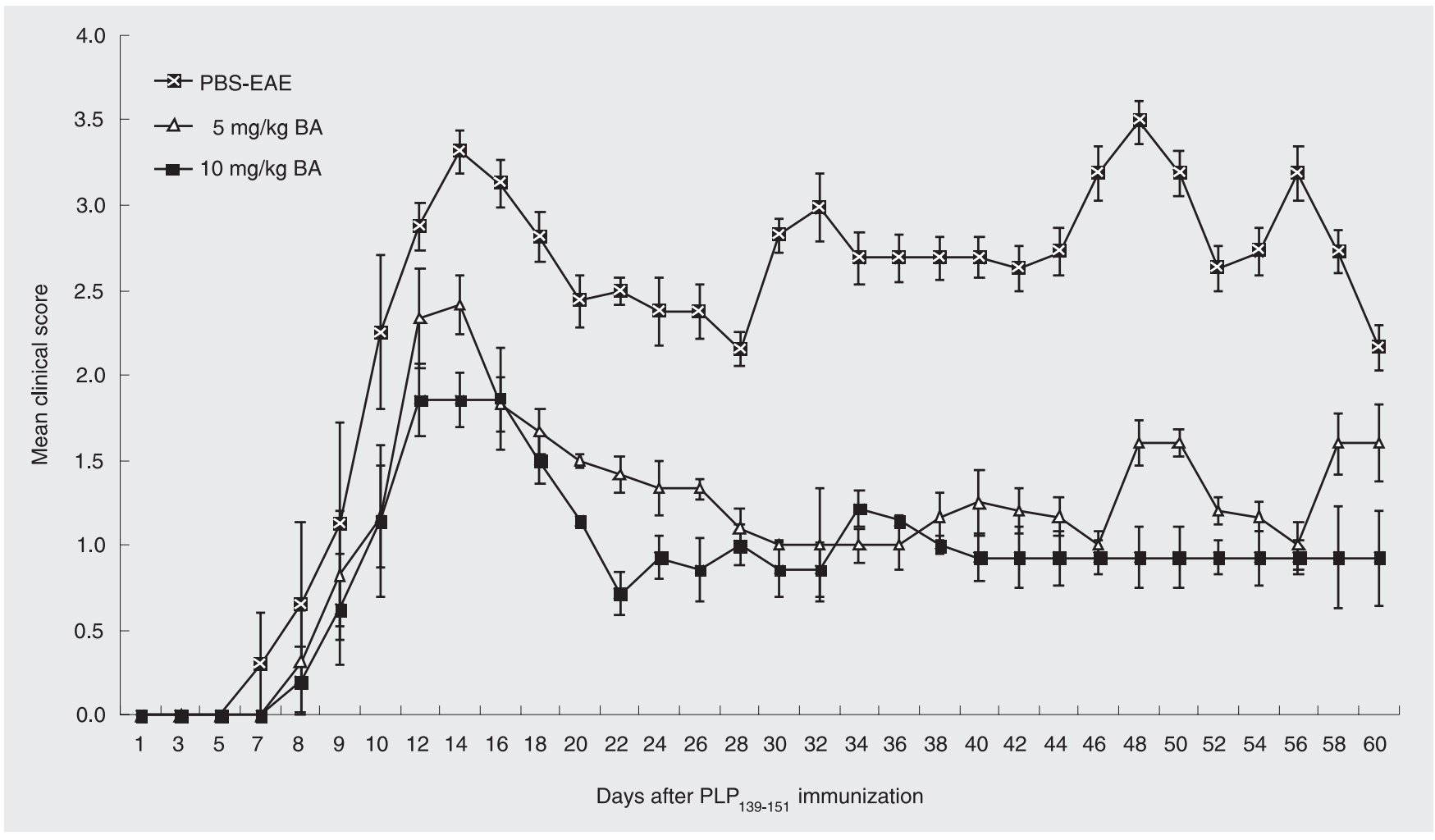

Figure 2. Effect of baicalin treatment on clinical score of experimental autoimmune encephalomyelitis associated disease activity. SJL/J female mice were immunized subcutaneously at three sites in the left flank with a total of $0.15 \mathrm{~mL}$ of an emulsion containing $150 \mathrm{mg} P \mathrm{PP}_{139-151}$ and an equal volume of complete Freund's adjuvant. Immediately and $48 \mathrm{~h}$ later, $300 \mathrm{ng}$ pertussis toxin was injected subcutaneously. Starting one day before immunization (day -1), the animals received a different dose of baicalin $(5$ or $10 \mathrm{mg} / \mathrm{kg}$ ) by intraperitoneal injection for three consecutive days. Animals were scored daily for clinical symptoms on a 0-6 scale. Mean clinical scores were determined for each treatment group $(\mathrm{N}=20)$. PBS-EAE = phosphate-buffered saline-experimental autoimmune encephalomyelitis; $\mathrm{BA}=$ baicalin; $\mathrm{PLP}_{139-151}=$ proteolipid protein 139-151. 
tion-dependent manner. There was a significant reduction in secreted IFN- $\gamma$ and a significant increase in secreted IL-4 (Figure 4B and C).

\section{Discussion}

EAE is an autoimmune disease of CNS mediated by $\mathrm{CD}^{+} \mathrm{T}$ lymphocytes specific for autoantigens of the myelin sheath, including myelin basic protein and proteolipid

Figure 3. Inflammation in a section of lumbar spinal cord from experimental autoimmune encephalomyelitis (EAE) + phosphate-buffered saline (PBS) and $\mathrm{EAE}$ + baicalin (BA; $10 \mathrm{mg} / \mathrm{kg}$ ) $\mathrm{SJL} / \mathrm{J}$ mice. BA reduced inflammation in the spinal cord of EAE. The lumbar region of the spinal cord was dissected from the animals ( $N=8$ per group) at the peak of clinical disease 14-20 days after immunization and fixed in $10 \%$ formalin. The spinal cords were embedded in paraffin and cut into 4- $\mu \mathrm{m}$ thick sections. The sections were stained with hematoxylin and eosin. $A$, Spinal cord from a PBS-treated mouse shows diffuse dense mononuclear infiltration surrounding vascellum and edge. $B$, Spinal cord from BA-treated mouse shows lesser dense mononuclear infiltration surrounding vascellum. C, The means and SEM of histologic scores of PBS- and BA-treated mice. Each cross-section was examined in each lumbar region of spinal cord. The data are summarized from each mouse in each group. ${ }^{*} \mathrm{P}=0.000$ vs $\mathrm{PBS}$ treated EAE (independent-sample $t$-test, $\mathrm{N}=8$ per group). Magnification: $100 \mathrm{X}$ for $\mathrm{A}$ and $\mathrm{B}$.

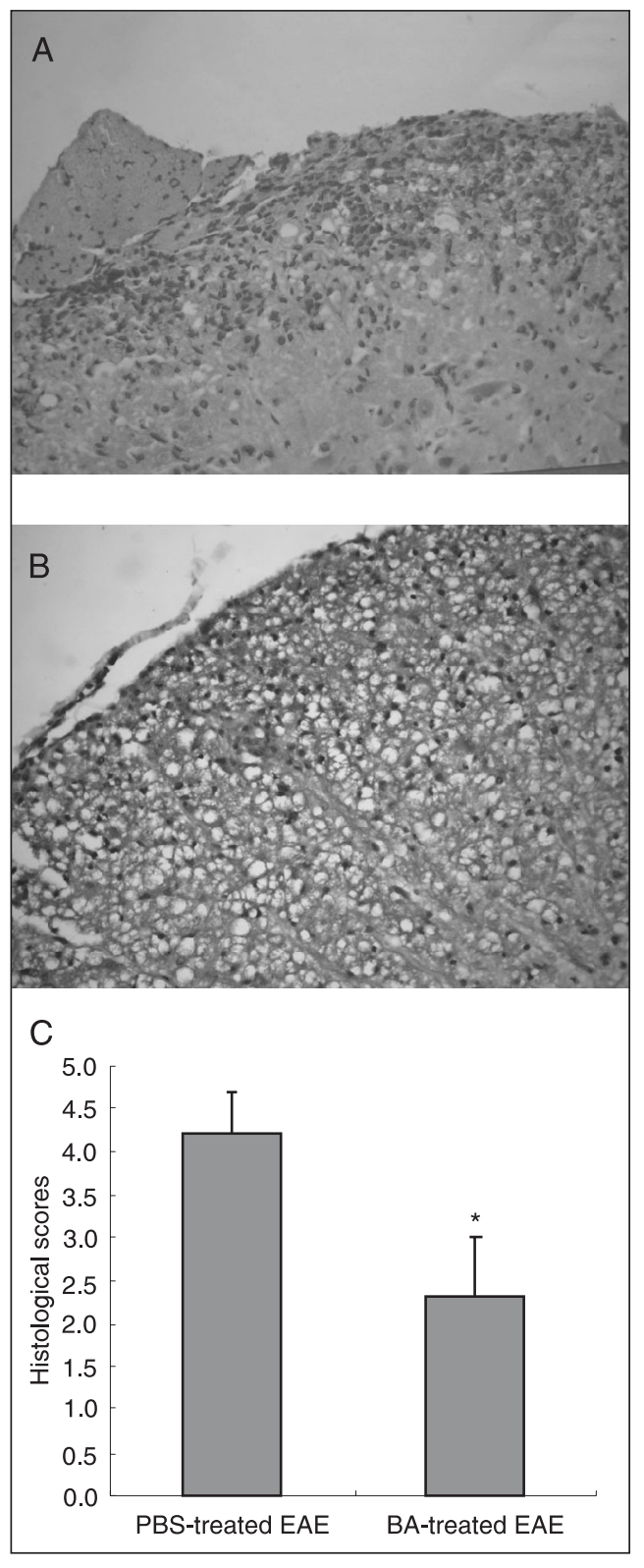

protein $(11,12)$. Remitting/relapsing EAE(RREAE) induced by active immunization with the immunodominant epitope of $\mathrm{PLP}_{139-151}$ (13), is characterized by an initial acute phase, followed by a series of remissions and relapses (14). CD4+ Th1 cells and their proinflammatory cytokines are suspected to be important in the pathogenesis of MS (15), and necessary for the induction of RR-EAE $(14,16,17)$. The cytokine profile and the nature of cellular infiltrate were markedly different during the clinical course of RR-EAE, wherein the maximal expression of the proinflammatory cytokines IFN- $\gamma$ and TNF- $\alpha$ are important mediators for disease induction. Th2 cell clones specific for encephalitogenic peptides are unable to induce the disease and can inhibit Th1 autoimmune clones, presumably by secreting IL-4, IL10 , and TGF- 3 (18-20). Several studies have suggested a significant role for IL-4, but not IL-10, in promoting RR-EAE remission.

Although we did not test directly the ability of BA to ameliorate disease symptoms at the peak of disease, our findings suggest that this drug is capable of suppressing a pre-activated immune system in the late effector phase leading into disease eruption. Since BA can penetrate into brain and spinal cord, we did not test the time point related to the stage of disease progression, in which the encephalitogenic T-cells are initiating infiltration into the CNS and beginning to recruit macrophages and microglia (21). The dose-response experiments carried out in the present study demonstrated that BA is therapeutic at the two doses used (Table 1). BA of $5 \mathrm{mg} / \mathrm{kg}$ is no less effective than that of $10 \mathrm{mg} / \mathrm{kg}$. Furthermore, the fact that no reversing trend observed in the amelioration of multiple disease parameters would suggest that the efficacy limit might be at $5 \mathrm{mg} /$ kg (Table 1, Figures 2 and 3).

The pathogenic processes in EAE may greatly rely on the secretion of IFN- $\gamma$ and IL-4, likely to be modulated by BA. Ex vivo studies using PILN isolated from EAE animals showed 
that BA $(5,15$, and $25 \mu \mathrm{M})$ inhibits the PILN MNC proliferation, as well as reduced IFN- $\gamma$ production while increasing IL-4 production in a concentration-dependent manner. These studies indicate that BA treatment inhibited a proinflammatory Th1-biased cytokine response as well as promoted an increase of antiinflammatory cytokine and attenuated clinical disease in the $\mathrm{PLP}_{139-151}$-induced model of $\mathrm{EAE}$ in SJL/J mice.

The dried roots of Scutellaria baicalensis Georgi (common name: Huangqin in China) have been widely employed for many centuries. They are effective against staphylococci, cholera, dysentery, pneumococci, and influenza virus. The active components are flavonoids, the major being BA, baicalein and wogonin (22). In our preliminary experiments, although all the three flavonoids manifested similar antioxidant activity, BA was more effective on antigen-specific inhibition of Th1 cells of autoimmune encephalomyelitis. The glycoside of baicalein, BA, is a potent anti-inflammatory and anti-tumor agent (23). BA showed strong anti-inflammatory activity, similar to that of dexamethasone in the treatment of endotoxic lung injury (5) and it could inhibit the binding of a number of chemokines to human leukocytes or cells transfected to express specific chemokine receptors (24) affecting cell migration. Recent studies showed that BA as a potential cancer chemopreventive agent inhibited T-cell proliferation and Th1 cytokines such as TNF- $\alpha$ and IFN- $\gamma(25)$. In the light of our observations and the literature findings that highlight the anti-inflammatory potential of $\mathrm{BA}$, we hypothesize that BA would alter the cytokine production pattern from Th1-type to favorable Th2-type and contribute to the reduced severity of EAE. The results obtained in this study clearly show that BA (5 and $10 \mathrm{mg} / \mathrm{kg}$ ) treatment effectively reduces the severity of EAE-associated clinical symptoms and neuroinflammation in spinal cord, and further show a differential cytokine response of PILN
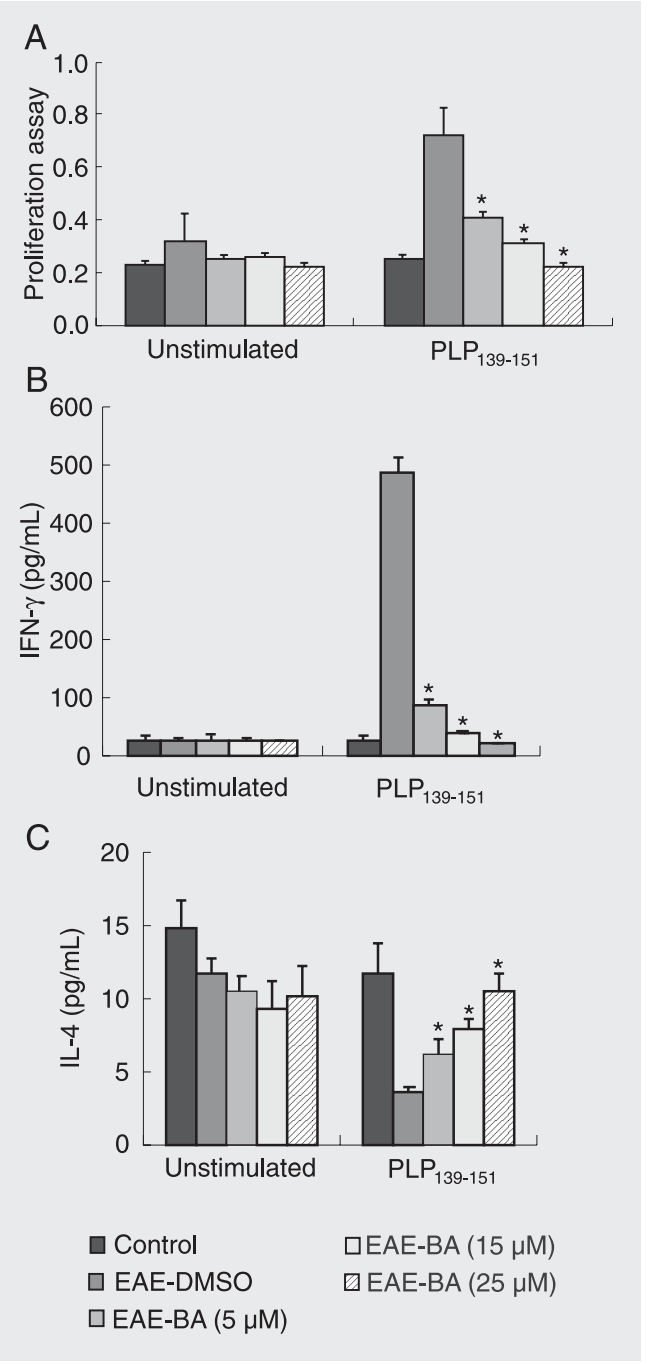

MNCs ex vivo, decreasing the proinflammatory Th1 cytokine IFN- $\gamma$ while increasing the anti-inflammatory Th2 cytokine IL- 4 . These results suggest that BA modulates EAE, at least in part, by suppressing the induction of IFN- $\gamma$ and increasing the induction of IL-4. This finding encourages further investigation on BA in its role as a potential candidate for developing more efficient therapy strategies against multiple sclerosis.

\section{Acknowledgments}

We would like to acknowledge Professor Qinwen JIN, Dr. Ting Wu and Dr. Qiu WEN for helpfull discussion with our experiments.
Figure 4. Effect of baicalin (BA) treatment on a proliferation assay (A) and on cytokine levels $(B, \mathrm{IFN}-\gamma$ and $C, \mathrm{IL}-4)$ in vitro. Animals with experimental autoimmune encephalomyelitis $(\mathrm{EAE})(\mathrm{N}=10)$ were analyzed at day 10 after immunization. Popliteal and inguinal lymph node mononuclear cells were stimulated with proteolipid protein 139-151 (PLP 139-151) and various concentrations of BA ( 5 , 15 , and $25 \mu \mathrm{M}$ ) or dimethylsulfoxide (DMSO) for $72 \mathrm{~h}$. Blank cells from control mice received an equivalent amount of DMSO. The proliferation assay was determined by MTT. The levels of IFN- $\gamma$ and IL-4 were determined by ELISA. ${ }^{*} \mathrm{P}<0.001$ vs EAEDMSO (one-way ANOVA followed by the post hoc multiple comparison Dunnett test). 


\section{References}

1. Zorzon M, Zivadinov R, Nasuelli D, Dolfini P, Bosco A, Bratina A, et al. Risk factors of multiple sclerosis: a case-control study. Neurol Sci 2003; 24: 242-247.

2. Taira Z, Yabe K, Hamaguchi Y, Hirayama K, Kishimoto M, Ishida S, et al. Effects of Sho-saiko-to extract and its components, Baicalin, baicalein, glycyrrhizin and glycyrrhetic acid, on pharmacokinetic behavior of salicylamide in carbon tetrachloride intoxicated rats. Food Chem Toxicol 2004; 42: 803-807.

3. Shimizu I, Ma YR, Mizobuchi Y, Liu F, Miura T, Nakai Y, et al. Effects of Sho-saiko-to, a Japanese herbal medicine, on hepatic fibrosis in rats. Hepatology 1999; 29: 149-160.

4. Ohta Y, Nishida K, Sasaki E, Kongo M, Hayashi T, Nagata M, et al. Comparative study of oral and parenteral administration of Shosaiko-to (xiao-chaihu-tang) extract on D-galactosamine-induced liver injury in rats. Am J Chin Med 1997; 25: 333-342.

5. Lo YC, Lin YL, Yu KL, Lai YH, Wu YC, Ann LM, et al. San-HuangXie-Xin-Tang attenuates inflammatory responses in lipopolysaccharide-exposed rat lungs. J Ethnopharmacol 2005; 101: 68-74.

6. Krakauer T, Li BQ, Young HA. The flavonoid baicalin inhibits superantigen-induced inflammatory cytokines and chemokines. FEBS Lett 2001; 500: 52-55.

7. De Rosa V, Procaccini C, La Cava A, Chieffi P, Nicoletti GF, Fontana $S$, et al. Leptin neutralization interferes with pathogenic $T$ cell autoreactivity in autoimmune encephalomyelitis. J Clin Invest 2006; 116 : 447-455.

8. Stosic-Grujicic S, Savic-Radojevic A, Maksimovic-Ivanic D, Markovic M, Bumbasirevic V, Ramic Z, et al. Down-regulation of experimental allergic encephalomyelitis in DA rats by tiazofurin. $J$ Neuroimmunol 2002; 130: 66-77.

9. Whitham RH, Bourdette DN, Hashim GA, Herndon RM, Ilg RC, Vandenbark AA, et al. Lymphocytes from SJL/J mice immunized with spinal cord respond selectively to a peptide of proteolipid protein and transfer relapsing demyelinating experimental autoimmune encephalomyelitis. J Immunol 1991; 146: 101-107.

10. Peng L, Wang B, Ren P. Reduction of MTT by flavonoids in the absence of cells. Colloids Surf B Biointerfaces 2005; 45: 108-111.

11. Liblau RS, Singer SM, McDevitt HO. Th1 and Th2 CD4+ T cells in the pathogenesis of organ-specific autoimmune diseases. Immunol Today 1995; 16: 34-38.

12. Ben-Nun A, Wekerle $H$, Cohen IR. The rapid isolation of clonable antigen-specific $\mathrm{T}$ lymphocyte lines capable of mediating autoimmune encephalomyelitis. Eur J Immunol 1981; 11: 195-199.

13. Kennedy MK, Tan LJ, Dal Canto MC, Tuohy VK, Lu ZJ, Trotter JL, et al. Inhibition of murine relapsing experimental autoimmune encepha- lomyelitis by immune tolerance to proteolipid protein and its encephalitogenic peptides. J Immunol 1990; 144: 909-915.

14. McRae BL, Kennedy MK, Tan LJ, Dal Canto MC, Picha KS, Miller SD. Induction of active and adoptive relapsing experimental autoimmune encephalomyelitis (EAE) using an encephalitogenic epitope of proteolipid protein. J Neuroimmunol 1992; 38: 229-240.

15. Beck J, Rondot P, Catinot L, Falcoff E, Kirchner H, Wietzerbin J. Increased production of interferon gamma and tumor necrosis factor precedes clinical manifestation in multiple sclerosis: do cytokines trigger off exacerbations? Acta Neurol Scand 1988; 78: 318-323.

16. Waldor MK, Sriram S, Hardy R, Herzenberg LA, Herzenberg LA, Lanier L, et al. Reversal of experimental allergic encephalomyelitis with monoclonal antibody to a T-cell subset marker. Science 1985; 227: 415-417.

17. McDonald AH, Swanborg RH. Antigen-specific inhibition of immune interferon production by suppressor cells of autoimmune encephalomyelitis. J Immunol 1988; 140: 1132-1138.

18. Racke MK, Bonomo A, Scott DE, Cannella B, Levine A, Raine CS, et al. Cytokine-induced immune deviation as a therapy for inflammatory autoimmune disease. J Exp Med 1994; 180: 1961-1966.

19. Miller A, al-Sabbagh A, Santos LM, Das MP, Weiner HL. Epitopes of myelin basic protein that trigger TGF-beta release after oral tolerization are distinct from encephalitogenic epitopes and mediate epitope-driven bystander suppression. J Immunol 1993; 151: 73077315.

20. Chen Y, Kuchroo VK, Inobe J, Hafler DA, Weiner HL. Regulatory T cell clones induced by oral tolerance: suppression of autoimmune encephalomyelitis. Science 1994; 265: 1237-1240.

21. Juedes $\mathrm{AE}$, Ruddle $\mathrm{NH}$. Resident and infiltrating central nervous system APCs regulate the emergence and resolution of experimental autoimmune encephalomyelitis. J Immunol 2001; 166: 51685175.

22. Zhao Y, Li H, Gao Z, Gong Y, Xu H. Effects of flavonoids extracted from Scutellaria baicalensis Georgi on hemin-nitrite- $\mathrm{H}_{2} \mathrm{O}_{2}$ induced liver injury. Eur J Pharmacol 2006; 536: 192-199.

23. Huang Y, Tsang SY, Yao X, Chen ZY. Biological properties of baicalein in cardiovascular system. Curr Drug Targets Cardiovasc Haematol Disord 2005; 5: 177-184.

24. Shen YC, Chiou WF, Chou YC, Chen CF. Mechanisms in mediating the anti-inflammatory effects of baicalin and baicalein in human leukocytes. Eur J Pharmacol 2003; 465: 171-181.

25. Li BQ, Fu T, Gong WH, Dunlop N, Kung H, Yan Y, et al. The flavonoid baicalin exhibits anti-inflammatory activity by binding to chemokines. Immunopharmacology 2000; 49: 295-306. 